\title{
Peak-to-Average Power Ratio analysis in MIMO systems
}

\author{
Basel Rihawi and Yves Louët \\ IETR/Supélec - Campus de Rennes \\ Avenue de la Boulaie, BP 81127, 35511 Cesson-Sévigné, France \\ Telephone: + (33)2 998445 00, Fax: +(33) 299844599 \\ E-mail : Yves.Louet,Basel.Rihawi@rennes.supelec.fr
}

\begin{abstract}
In this paper, we analyze the Peak-to-Average Power Ratio (PAPR) in Multiple Input Multiple Output (MIMO) systems based on Alamouti diversity scheme. This analysis is at both transmitter and receiver sides with channel influences. By giving mathematical formulations of the Alamouti scheme signals, we give an upper bound of this signal when Nyquist shaping (raised cosine) filter is considered with ideal channel. The PAPR is derived for Binary Phase-Shift Keying (BPSK). Furthermore, we analyze the Additive White Gaussian Noise (AWGN) influence on the Cumulative Distribution Function (CDF) of PAPR of a received signal when a deterministic signal is transmitted.
\end{abstract}

\section{Introduction}

Multiple-Input Multiple-Output (MIMO) has attracted considerable interest and has been studied intensively in the recent years due to their potential to increase the channel capacity in fading channels [1]. It has been shown that MIMO systems can support higher data rates under the same transmit power budget and bit-error-rate performance requirements as a single-input single-output (SISO) system. Multiple antennas at basestations and subscribers in a cellular system generate a sum of several signals at every reception antenna. This signal needs to be amplified by a Low Noise Amplifier (LNA). Unfortunately, the nonlinear characteristics of the LNA and the nonconstant envelope of the received signal (i.e. large PAPR) generate nonlinear distortions and intermodulations [2]. This paper is organized as follows: section 2 describes some notions and measures related to PAPR-single carrier in SISO system. Section 3 describes MIMO systems based on Alamouti diversity schemes which we have used in this study. The main idea of this paper is discussed in section 4, where we study the PAPR in MIMO-Alamouti without the effects of channel and we present some simulation results with presence and non presence of AWGN. In Section 5, we analyze the CDF of PAPR of a deterministic signal in case of SISO system.

\section{PAPR and PMEPR definitions for SISO-single carrier}

The peak factor of a signal $S(t)$ is an important attribute of a communication system. It is a measure of the amplitude fluctuations of the signal and is usually defined as the ratio between the maximum instantaneous power that $S(t)$ might reach, and its average power [3]. In the literature, Peak-to-Mean Envelop Power Ratio (PMEPR) and PAPR are sometimes used interchangeably and we have to know that they are parameters that depends on the peak of the signal over the whole time interval, and not just on its samples. Moreover, for a large number of samples per symbol (more than Nyquist frequency sampling) and large constellation size, the computational effort in finding PAPR and PMEPR will become large [4].

\subsection{Definition of PMEPR}

The PMEPR is used when the basedband signal is considered (complex envelope) $\widetilde{S}(t)=S_{I}(t)+j S_{Q}(t)$, [5]. The mathematical expression of PMEPR is :

$$
\operatorname{PMEPR}\{\widetilde{S}(t)\}=\frac{\operatorname{Max}|\widetilde{S}(t)|^{2}}{E\left\{|\widetilde{S}(t)|^{2}\right\}},
$$

where the variable $t$ is real and $E\{$.$\} denotes the ex-$ pectation (statistical averaging) operation.

\subsection{Definition of PAPR}

The PAPR is commonly defined as a derivation for Radio-Frequency ( $\mathrm{RF}$ ) signals. It can be written by :

$$
\operatorname{PAPR}\{\widetilde{S}(t)\}=\frac{\operatorname{Max}\left|\operatorname{Re}\left(\widetilde{S}(t) e^{j 2 \pi f_{c} t}\right)\right|^{2}}{E\left\{\left|\operatorname{Re}\left(\widetilde{S}(t) e^{j 2 \pi f_{c} t}\right)\right|^{2}\right\}},
$$

where $f_{c}$ is the carrier frequency.

The PAPR is a measure of the signal fluctuations after the RF transposition (so just before the power amplifier device).

\subsection{The PMEPR and PAPR computa- tions for Raised Cosine filter}

After the definitions in the last subsections, we will compute the PMEPR or PAPR at the different stages at the transmitter-side of communication system shown in Figure 1.

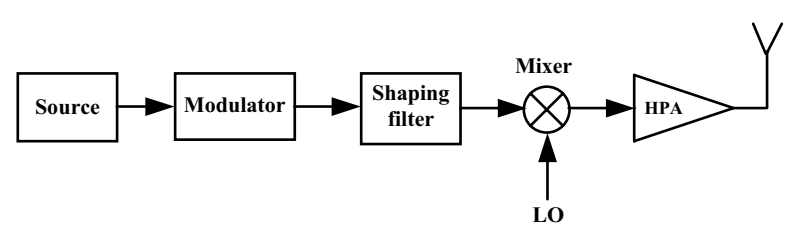

Figure 1. Transmitter circuit blocks

The shaping filter used is a Raised Cosine (RC) 
filter which has an impulse response $p(t)$ :

$$
p(t)=\frac{\sin \left(\frac{\pi}{T_{s}} t\right)}{\frac{\pi}{T_{s}}} \frac{\cos \left(\frac{\pi \beta}{T_{s}} t\right)}{1-\frac{4 \beta^{2} t^{2}}{T_{s}^{2}}},
$$

where $\beta$ is the filter roll-off factor and $T_{s}$ is the symbol period.

In the case of a BPSK modulation, the PMEPR at the output of the shaping filter is upper bounded by [5] :

$$
P M E P R\{\tilde{S}(t)\} \leq \frac{\pi^{2} / 8 \beta}{(1-\beta / 4)} .
$$

In the case of a QAM modulation, the PMEPR at the output of the $\mathrm{RC}$ filter become :

$$
\operatorname{PMEPR}\{\tilde{S}(t)\} \leq\left(\frac{\operatorname{Max}\left(a_{k}\right)}{\sigma_{a}}\right)^{2} \frac{\pi^{2} / 8 \beta}{(1-\beta / 4)} .
$$

And the PAPR of the passband signal $\operatorname{Re}\left\{\widetilde{S}(t) e^{j 2 \pi f_{c} t}\right\}$ (after the mixer which used to up conversion the baseband to a radio frequency signal) is [6] :

$$
P A P R_{d B} \approx P M E P R_{d B}+3 d B .
$$

This last relationship between PAPR and PMEPR is conditioned by $f_{c}>B$ (where $\mathrm{B}$ is the bandwidth of signal), what is the case in most practical systems. It is clear that from (6), the passband PAPR is approximately twice (3dB higher) than the baseband PMEPR.

In general, we can compute the PMEPR of the modulated signal at the output of the raised cosine filter, by summing the PMEPR (in $\mathrm{dB}$ ) of modulated signal with a factor $f(\beta)=\frac{\pi^{2} / 8 \beta}{(1-\beta / 4)}$ (in $\left.\mathrm{dB}\right)$ which represents the RC influence on the PMEPR. In the case of PSK, the PMEPR is unity, so there is no contribution. For QAM modulation, the PMEPR contribution depends on the order of the modulation. The PMEPR of a constellation is computed by assuming each symbol is equally likely. Thus, the PMEPR is computed by dividing the magnitude squared of the largest symbol by the average of the square of each of the symbols [7]. Table 1 shows the PMEPR of several constellations expressed in $\mathrm{dB}$. It is interesting to note that the PMEPR of 16-QAM is greater than that of 32-QAM. This is because 16-QAM is a square constellation, while the 32-QAM constellation is nearly round. Table 2 shows a comparison of the

Table 1. PMEPR of the constellations for different modulation schemes

\begin{tabular}{|c|c|}
\hline Constellation & PMEPR \\
\hline \hline N-PSK & $0 \mathrm{~dB}$ \\
16-QAM & $2.6 \mathrm{~dB}$ \\
32-QAM & $2.3 \mathrm{~dB}$ \\
64-QAM & $3.7 \mathrm{~dB}$ \\
128-QAM & $4.3 \mathrm{~dB}$ \\
\hline
\end{tabular}

factor $f(\beta)$ of the RC filter for several different values of $\beta$. Thus, table 1 and 2 provide all of the data required for determining the maximum PMEPR of a given signal.
Table 2. PMEPR of the RC filter for different $\beta$

\begin{tabular}{|c|c|}
\hline$\beta$ & $f(\beta)$ \\
\hline \hline 0.15 & $9.3 \mathrm{~dB}$ \\
0.2 & $8.1 \mathrm{~dB}$ \\
0.3 & $6.5 \mathrm{~dB}$ \\
0.4 & $5.4 \mathrm{~dB}$ \\
0.5 & $4.5 \mathrm{~dB}$ \\
\hline
\end{tabular}

\section{MIMO -Alamouti coding scheme}

MIMO stands for Multiple Input Multiple Output and most often refers to the multiplicity of antennas at the transmitter and the receiver side. There are different schemes of MIMO. We have studied one of these schemes, which is proposed by Siavash M. Alamouti [8]. This scheme has the advantage of achieving a high spatial diversity order in the absence of channel knowledge at the transmitter while keeping the number of receive antennas at the mobile set to a small number. We have concentrated on a two transmitters and two receivers configuration scheme with the channel model depicted in Figure 2.

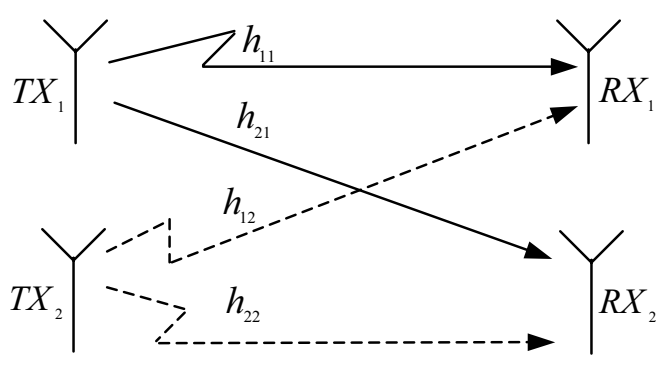

Figure 2. Channel Model for MIMO $2 \times 2$

$h_{11}, h_{12}, h_{21}, h_{22}$ denotes the channels impulses responses between the transmit and receive antennas.

In the Alamouti MIMO scheme [8], diversity is introduced both in space and time to combat the effects of time-varying multipath fading. The diversity is achieved using the Alamouti Space Time Coding where the signal is coded at the transmitter and decoded in the receiver. The symbols, $c_{0}$ and $c_{1}$ are

\begin{tabular}{|c|c|c|}
\hline \multicolumn{3}{|c|}{ tem } \\
\hline & $T X_{1}$ & $T X_{2}$ \\
\hline time:t & $c_{0}$ & $c_{1}$ \\
\hline time:t+T & $-c_{1}^{*}$ & $c_{0}^{*}$ \\
\hline
\end{tabular}
coded at the transmitter according to table 3 :

Table 3 . Alamouti Space-Time Coding for $2 \times 2 \mathrm{MIMO}$ system

$c^{*}$ denotes the complex conjugate of $c$.

Using this coding scheme at the transmitter, the received signals $r_{1}$ and $r_{2}$ at the receiver antennas $R X_{1}$ and $R X_{2}$ will be respectively :

$$
\begin{gathered}
r_{1}(t)=h_{11} c_{0}+h_{12} c_{1}+n_{1}, \\
r_{1}(t+T)=-h_{11} c_{1}^{*}+h_{12} c_{0}^{*}+n_{1}^{\prime},
\end{gathered}
$$




$$
\begin{gathered}
r_{2}(t)=h_{21} c_{0}+h_{22} c_{1}+n_{2}, \\
r_{2}(t+T)=-h_{21} c_{1}^{*}+h_{22} c_{0}^{*}+n_{2}^{\prime},
\end{gathered}
$$

where $n_{1}, n_{1}^{\prime}, n_{2}$ and $n_{2}^{\prime}$ represent the noise channel. The received signals are combined as :

$\tilde{c}_{0}=h_{11}^{*} r_{1}(t)+h_{12} r_{1}^{*}(t+T)+h_{21}^{*} r_{2}(t)+h_{22} r_{2}^{*}(t+T)$, $\tilde{c}_{1}=h_{12}^{*} r_{1}(t)-h_{11} r_{1}^{*}(t+T)+h_{22}^{*} r_{2}(t)-h_{21} r_{2}^{*}(t+T)$, $\tilde{c}_{0}$ and $\tilde{c}_{1}$ are sent to the detector.

Table 4. Notation for received signals

\begin{tabular}{|c|c|c|}
\hline & $R X_{1}$ & $R X_{2}$ \\
\hline \hline time:t & $r_{1}(t)$ & $r_{2}(t)$ \\
\hline time:t+T & $r_{1}(t+T)$ & $r_{2}(t+T)$ \\
\hline
\end{tabular}

\section{PAPR of MIMO-Alamouti with ideal channel}

The communication system using the Alamouti model is depicted in Figure 3.

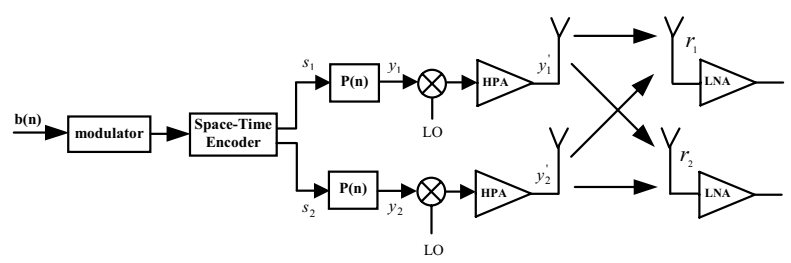

Figure 3. Communication system using the Alamouti scheme

From the table 3 , we can derive the signals at the output of the space-time encoder as :

$$
\begin{aligned}
& s_{1}(t)=\sum_{l=0}^{\frac{N}{2}-1}\left[c_{2 l} \delta(t-2 l T)-c_{2 l+1}^{*} \delta(t-(2 l+1) T)\right], \\
& s_{2}(t)=\sum_{l=0}^{\frac{N}{2}-1}\left[c_{2 l+1} \delta(t-2 l T)+c_{2 l}^{*} \delta(t-(2 l+1) T)\right],
\end{aligned}
$$

where $\delta(t)$ denotes an impulse at $t=0$.

By taking into account the filtering influence,

$$
\begin{aligned}
y_{1}(t) & =\sum_{k=0}^{\frac{N}{2}-1}\left[c_{2 k} p(t-2 k T)-c_{2 k+1}^{*} p(t-(2 k+1) T)\right], \\
y_{2}(t) & =\sum_{k=0}^{\frac{N}{2}-1}\left[c_{2 k+1} p(t-2 k T)+c_{2 k}^{*} p(t-(2 k+1) T)\right],
\end{aligned}
$$

where $k$ is the index for the sample at time $k T$.

Now, let us analyze the signal at one of the receiver antenna by considering a linear amplifier at the transmitter with gain $G=1$ and an ideal channel as :

$$
r_{1}(t)=y_{1}(t) e^{j \omega_{c} t}+y_{2}(t) e^{j \omega_{c} t},
$$

$$
\begin{aligned}
r_{1}(t) & =\sum_{k=0}^{\frac{N}{2}-1}\left[\left(c_{2 k}+c_{2 k+1)} p(t-2 k T)\right.\right. \\
& \left.+\left(c_{2 k}^{*}-c_{2 k+1}^{*}\right) p(t-(2 k+1))\right] e^{j \omega_{c} t} .
\end{aligned}
$$

We finally get :

$$
r_{1}(t)=\sum_{k=0}^{N-1} \alpha_{k} p\left(t-k T_{s}\right) e^{j \omega_{c} t},
$$

where :

$$
\alpha_{k}= \begin{cases}c_{k}+c_{k+1} & \text { if } k \text { even } \\ c_{k}^{*}-c_{k+1}^{*} & \text { if } k \text { odd }\end{cases}
$$

From (13), we return from a MIMO-Alamouti case to a SISO case.

Using Eqs.(4) and (6), the PAPR at the antenna-1 at the receiver side (at the input of LNA) can be upper bounded by :

$$
P A P R\left\{r_{1}(t)\right\} \leq\left(\frac{\operatorname{Max}\left(\alpha_{k}\right)}{\sigma_{\alpha}}\right)^{2} \frac{\pi^{2} / 8 \beta}{(1-\beta / 4)}+3_{d B} .
$$

Here, the $\alpha_{k}$ has the following characteristics in the case of a BPSK modulation :

$$
\left\{\begin{array}{l}
\operatorname{Pr}\left[\alpha_{k}=+2\right]=0.25 \\
\operatorname{Pr}\left[\alpha_{k}=0\right]=0.5 \\
\operatorname{Pr}\left[\alpha_{k}=-2\right]=0.25
\end{array}\right.
$$

Then,

The maximal value of $\alpha_{k}$ is :

$$
\operatorname{Max}\left(\alpha_{k}\right)=2 \text {. }
$$

The expected-square or mean-square value of a $\alpha_{k}$ is

$$
\sigma_{\alpha}^{2}=\sum_{i=1}^{3} \alpha_{i}^{2} p\left(\alpha_{i}\right)
$$

where $p\left(\alpha_{i}\right)$ is the probability of $\alpha_{i}$ occurring,

$$
\sigma_{\alpha}^{2}=\frac{1}{4}(+2)^{2}+\frac{1}{2}(0)^{2}+\frac{1}{4}(-2)^{2}=2
$$

Finally, we get :

$$
\left(\frac{\operatorname{Max}\left(\alpha_{k}\right)}{\sigma_{k}}\right)^{2}=\frac{4}{2}=2 .
$$

The PAPR of the signal $r_{2}(t)$ can be expressed similarly as (14).

\subsection{Results and simulation}

We use computer simulations to evaluate the performances of the PAPR computation. We set the oversampling factor $L=4$ which is enough to detect the presence of peaks with a satisfactory precision [9]. Figure 4 shows the PAPR of received signal $r_{1}(t)$ as a function of roll off factor $(\beta)$ of the RC filter. For the $\beta$ values range up to about 0.6 , the computation has been led on the base of $10^{4}$ loops of $\mathrm{N}=1000$ symbols each to get the maximum PAPR value. It can be seen that the similitude between the theory 
and simulation is good. For the remain of $\beta$ ranges (greater than 0 and less than 0.6), we have $10^{5}$ loops of $N=10^{4}$ symbols each. This can be explained in part because of the statistical comportment of the infinite PAPR : when $\beta$ tends to zero, we have observe that the PAPR probability density function is dispersive and getting the infinite PAPR value needs large computations whereas, for larger roll off factor values, the probability law is more concentrated.

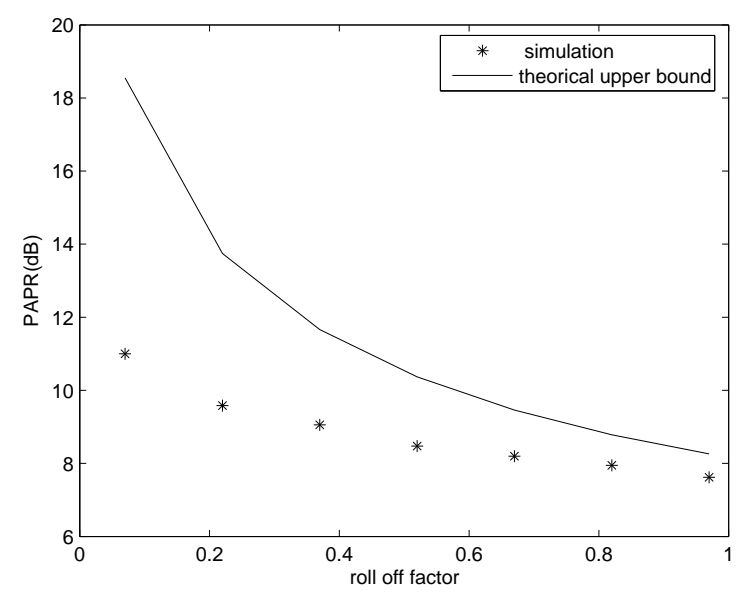

Figure 4. PAPR vs. roll off factor

\section{PAPR analysis with AWGN channel}

\subsection{Theoretical analysis in the case of a deterministic signal}

Let $S_{e}(t)$ be a deterministic signal. When such a signal is transmitted over an AWGN channel $N(t)$, the received signal can be expressed as :

$$
S_{r}(t)=S_{e}(t)+N(t) .
$$

This noise is a white Gaussian random process with mean zero, flat power spectral density $\frac{N_{0}}{2}$ and probability density function defined as :

$$
f_{x}(x)=\frac{1}{\sqrt{2 \pi \sigma^{2}}} e^{-\frac{x^{2}}{2 \sigma^{2}}}
$$

Since the received signal follows a gaussian distribution, thus it has the possibilities of infinitely high levels. In these cases, instead of derive the PAPR value, one may consider the probability of exceeding a certain level.

The aim of our study is to analyse the CDF of the PAPR of the received signal at the input of LNA.

To simplify this study, let us deal with the discrete signal.

The discrete-time representation of Eq.15 is :

$$
S_{r}(n)=S_{e}(n)+N(n), \quad n \in[0, N-1]
$$

The probability density function $f_{y}(y)$ of $Y=\frac{S_{r}^{2}}{P_{r}}$, where $P_{r}$ is the mean power of $S_{r}$ is :

$$
f_{y}(y)=\frac{\sqrt{P_{r}}}{2 \sqrt{y}}\left(f_{s_{r}}\left(\sqrt{P_{r} y}\right)+f_{s_{r}}\left(-\sqrt{P_{r} y}\right)\right. \text {. }
$$

Then, from (16) and (18), we get :

$$
\begin{aligned}
f_{y}(y) & =\frac{\sqrt{P_{r}}}{2 \sqrt{y}}\left(\frac{1}{\sqrt{2 \pi \sigma_{b}}} e^{-\frac{\left(\sqrt{P_{r} y}-s_{e}\right)^{2}}{2 \sigma_{b}^{2}}}\right. \\
& \left.+\frac{1}{\sqrt{2 \pi \sigma_{b}}} e^{-\frac{\left(-\sqrt{P_{r} y}-s_{e}\right)^{2}}{2 \sigma_{b}^{2}}}\right) .
\end{aligned}
$$

The CDF of the PAPR can be expressed by :

$$
\operatorname{Pr}\left[\frac{\left|s_{r}(n)\right|^{2}}{P_{r}} \leq \gamma_{0}\right]=\int_{0}^{\gamma_{0}} f_{y}(y) d y .
$$

Then,

$$
\begin{array}{r}
\operatorname{Pr}\left[\frac{\left|s_{r}(n)\right|^{2}}{P_{r}} \leq \gamma_{0}\right]=\frac{\sqrt{P_{r}}}{2 \sqrt{2 \pi} \sigma_{b}} \\
{\left[\int _ { 0 } ^ { \gamma _ { 0 } } \left(\frac{1}{\sqrt{P_{r}}} e^{-\frac{\left(\sqrt{P_{r} y}-s_{e}(n)\right)^{2}}{2 \sigma_{b}^{2}}}\right.\right.} \\
\left.\left.+\frac{1}{\sqrt{P_{r}}} e^{-\frac{\left(-\sqrt{P_{r} y}-s_{e}(n)\right)^{2}}{2 \sigma_{b}^{2}}}\right) d y\right] .
\end{array}
$$

From last equation,

$$
\begin{aligned}
\operatorname{Pr}\left[\frac{\left|s_{r}(n)\right|^{2}}{P_{r}} \leq \gamma_{0}\right]= \\
\quad \frac{1}{2} \operatorname{erf}\left(\frac{\sqrt{P_{r}} \sqrt{\gamma_{0}}-s_{e}(n)}{\sqrt{2} \sigma_{b}}\right) \\
\quad+\frac{1}{2} \operatorname{erf}\left(\frac{\sqrt{P_{r}} \sqrt{\gamma_{0}}+s_{e}(n)}{\sqrt{2} \sigma_{b}}\right),
\end{aligned}
$$

where $\operatorname{erf}($.$) denotes the error function, defind as :$

$$
\operatorname{erf}(x)=\frac{2}{\sqrt{\pi}} \int_{0}^{x} e^{-t^{2}} d t
$$

Let us denote the data of length $N$ as a vector $S_{r}=\left[s_{r}(0), s_{r}(1), \ldots, s_{r}(N-1)\right]$ whose scalers are independent. The CDF that non of them exceed $\gamma_{0}$ is given by :

$$
\begin{aligned}
& \operatorname{Pr}\left[\frac{\max _{n<(N-1)}\left|s_{r}(n)\right|^{2}}{P_{r}} \leq \gamma_{0}\right]= \\
& \prod_{n=0}^{N-1}\left[\frac{1}{2} \operatorname{erf}\left(\frac{\sqrt{P_{r}} \sqrt{\gamma_{0}}-s_{e}(n)}{\sqrt{2} \sigma_{b}}\right)\right. \\
& \left.+\frac{1}{2} \operatorname{erf}\left(\frac{\sqrt{P_{r}} \sqrt{\gamma_{0}}+s_{e}(n)}{\sqrt{2} \sigma_{b}}\right)\right] .
\end{aligned}
$$

We can simplify this last equation by using the following convexity characteristic of error function :

$$
\operatorname{erf}(x+y)+\operatorname{erf}(x-y) \leq 2 \operatorname{erf}(x) \quad \forall x \geq 0 \quad \forall y
$$

Then, the Eq.23 becomes :

$$
\begin{gathered}
\operatorname{Pr}\left[\frac{\max _{0 \leq n<(N-1)}\left|s_{r}(n)\right|^{2}}{P_{r}} \leq \gamma_{0}\right] \leq \\
{\left[\operatorname{erf}\left(\frac{\sqrt{P_{r}} \sqrt{\gamma_{0}}}{\sqrt{2} \sigma_{b}}\right)\right]^{N} .}
\end{gathered}
$$

Figure 5 shows this CDF when a sinus signal is used as a deterministic signal. 


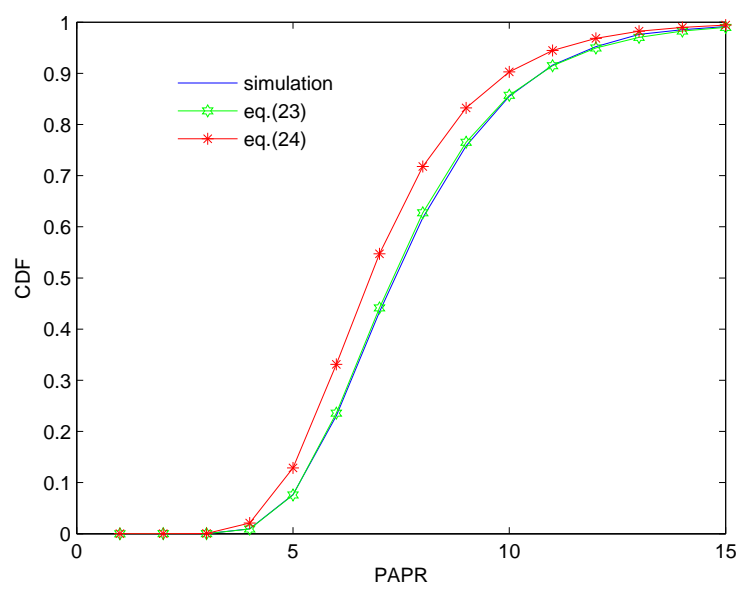

Figure 5. CDF of PAPR of deterministic signal over AWGN channel, $\mathrm{N}=1000$

\subsection{Case of MIMO-Alamouti}

In this section, we give a simulation result of $\mathrm{CDF}$ of PAPR of received signal studied in the section 4 in the presence of AWGN. Figure 6 is a plot of this CDF for different values of signal-to-noise ratio(SNR).

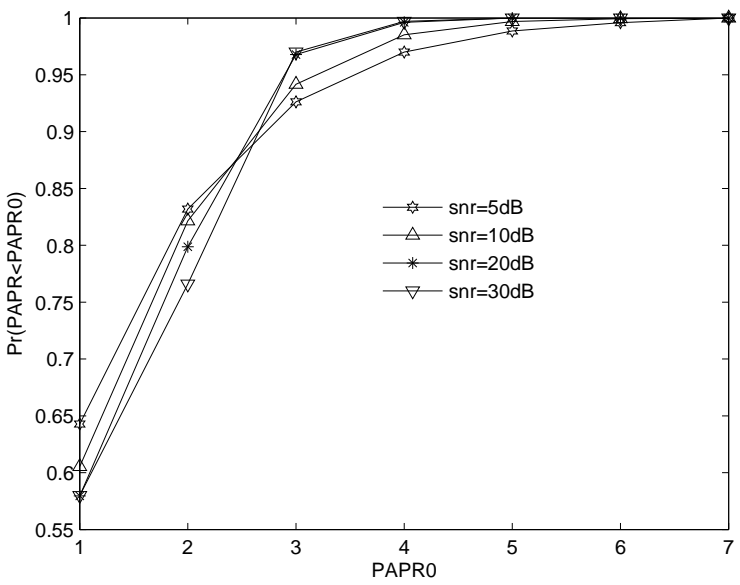

Figure 6. CDF of PAPR of Alamouti received signal for various values of SNR

\section{Conclusion}

In this paper, we have analyzed the MIMO systems based on Alamouti diversity scheme and we have derived the PMEPR and PAPR of a single carrier signal at the different stages of the transmitter side and the PAPR vs. the roll off factor of a RC filter and vs. SNR at the input of LNA in the receiver side. The channel model under consideration is an ideal channel. For AWGN channel, we have analyzed the CDF of PAPR of a deterministic signal in case of a SISO system, and finally, we have given a simulation of this $\mathrm{CDF}$ in the case of MIMO-Alamouti.

\section{References}

[1] A. Paulraj, R. Nabar, and D. Gore, Introduction to Space-Time Wireless Communications, preprint, Cambridge University Press, Cambridge, UK, 2003.

[2] Mattias Wennstrom, "On MIMO Systems and adaptive arrays for Wireless communica-tion", Uppsala University, 2002.

[3] Nati Dinur and Dov wulich, "Peak-to-Average Power Ratio in High-Order OFDM", IEEE Transactions on Communications, vol. 49, no. 6 , June 2001.

[4] S. Shepherd, J. Orriss, S. Barton, "Asymptotic limits in peak envelope power reduction by redundant coding on orthogonal frequency division miltiplex modulaion," IEEE Trans. On Comm., vol.46, no. 1, pp. 510, january 1998.

[5] Jacques Palicot, Yves Louët, "Power Ratio definitions and analysis in single carrier modulation", EUSIPCO 2005, Antalya, Turkey.

[6] Yves Louët, Jacques Palicot, "Synthèse de la notion de facteur de crte et application aux modulations monoporteuse", Gretsi 2005, Louvain-laNeuve- Belgique.

[7] Fuqin Xiong, "Digital Modulation Techniques", Artech House, 2000, pg 430.

[8] Siavash M. Alamouti, "A simple Transmit Diversity Technique for Wireless communications", IEEE Journal on Select Areas in Communications, Vol. 16, no. 8, October 1998.

[9] J. Tllado, "Peak to Average Power reduction for Multicarrier Modulation", Ph.D. Thesis Dissertation, Stanford University, September 1999.

[10] David Tse, "Fundamentals of Wireless Communication", University of California, Berkeley Pramod Viswanath, University of Illinois, Urbana-Champaign July 5, 2004 